\title{
UNIVALENCY OF SOME OPERATORS FOR ANALYTIC FUNCTIONS
}

\author{
S. Owa, H. Saitoh, J. Sokóe, M. Nunokawa
}

Abstract. For analytic functions $f(z)$ in the open unit disk $\mathbb{U}$, univalency of some integral operators concerning with Alexander type integrals is considered. Also some subordinations for analytic functions $f(z)$ in $\mathbb{U}$ are discussed with the Schwarzian derivative of $f(z)$.

2010 Mathematics Subject Classification: 30C45, 30C80.

Keywords: Schwarzian derivative, Bazilević function, close-to-convex function, subordination, convex function.

\section{INTRODUCTION}

Let $\mathcal{H}$ denote the class of functions $f(z)$ which are analytic in the open unit disk $\mathbb{U}=\{z \in \mathbb{C}:|z|<1\}$. Also let $\mathcal{A}$ be the subclass of $\mathcal{H}$ consisting of functions $f(z)$ of the form

$$
f(z)=z+\sum_{n=2}^{\infty} a_{n} z^{n} \quad(z \in \mathbb{U}) .
$$

Let $\mathcal{S}$ be the subclass of $\mathcal{A}$ consisting of $f(z)$ which are univalent in $\mathbb{U}$. If $f(z) \in \mathcal{A}$ satisfies

$$
\operatorname{Re}\left(\frac{z f^{\prime}(z)}{f(z)}\right)>\alpha \quad(z \in \mathbb{U})
$$

for some real $\alpha(0 \leqq \alpha<1)$, then $f(z)$ is said to be starlike of order $\alpha$ in $\mathbb{U}$ and denoted by $f(z) \in \mathcal{S}^{*}(\alpha)$. For $\alpha=0$, we say that $f(z) \in \mathcal{S}^{*}$ is starlike with respect to the origin. Further, if a fnction $f(z) \in \mathcal{A}$ satisfies $z f^{\prime}(z) \in \mathcal{S}^{*}(\alpha)(0 \leqq \alpha<1)$, then $f(z)$ is said to be convex of order $\alpha$ in $\mathbb{U}$ and denoted by $f(z) \in \mathcal{K}(\alpha)$. A function $f(z) \in \mathcal{K}(\alpha)$ satisfies

$$
\operatorname{Re}\left(1+\frac{z f^{\prime \prime}(z)}{f^{\prime}(z)}\right)>\alpha \quad(z \in \mathbb{U}) .
$$


S. Owa, H. Saitoh, J. Sokół, M. Nunokawa - Univalency of some operators ...

For $\alpha=0$, we write that $\mathcal{K}(0) \equiv \mathcal{K}$. We note that

$$
\mathcal{K}(\alpha) \subset \mathcal{S}^{*}(\alpha) \subset \mathcal{S} \subset \mathcal{A} \subset \mathcal{H}
$$

If there exists a function $g(z) \in \mathcal{K}$ such that

$$
\operatorname{Re}\left(e^{-i \beta} \frac{f^{\prime}(z)}{g(z)}\right)>0 \quad(z \in \mathbb{U})
$$

for $\beta \in(-\pi / 2, \pi / 2)$ and $f(z) \in \mathcal{A}$, then $f(z)$ is said to be close-to-convex in $\mathbb{U}$ and denoted by $f(z) \in \mathcal{C}$. It is known that $\mathcal{C} \subset \mathcal{S}$.

For $f(z) \in \mathcal{H}$, the Schwarzian derivative of $f(z)$ is given by

$$
\{f ; z\}=6\left(\frac{\partial^{2}}{\partial z \partial \zeta} \log \left(\frac{f(z)-f(\zeta)}{z-\zeta}\right)\right)_{z=\zeta}=\left(\frac{f^{\prime \prime}(z)}{f^{\prime}(z)}\right)^{\prime}-\frac{1}{2}\left(\frac{f^{\prime \prime}(z)}{f^{\prime}(z)}\right)^{2} .
$$

For the Schwarzian derivative $\{f ; z\}$ for $f(z) \in \mathcal{H}$, it is well-known that if $f(z) \in \mathcal{H}$ is univalent in $\mathbb{U}$, then

$$
|\{f ; z\}| \leqq \frac{6}{\left(1-|z|^{2}\right)^{2}} \quad(z \in \mathbb{U})
$$

and the equality holds true for the Koebe function $f(z)=z /(1-z)^{2}$. Further, we know that the Nehari's condition (see Nehari [10])

$$
|\{f ; z\}| \leqq \frac{2}{\left(1-|z|^{2}\right)^{2}} \quad(z \in \mathbb{U})
$$

implies that $f(z) \in \mathcal{H}$ is univalent in $\mathbb{U}$.

Note that $f(z) \in \mathcal{A}$ is uniformly locally univalent if and only if the pre-Schwarzian derivative

$$
T_{f}(z)=\frac{f^{\prime \prime}(z)}{f^{\prime}(z)}
$$

is hyperbolically bounded, that is, that the norm

$$
\|f\|=\sup _{|z|<1}\left(1-|z|^{2}\right)\left|T_{f}(z)\right|
$$

is finite. This quantity can be regarded as the Bloch norm of function $(\log f(z))^{\prime}$. Both of the pre-Schwarzian derivative and the norm $\|f\|$ play a central role in the theory of Teichmüller spaces, inner radius of univalence, quasiconformal extension, etc.. If $f(z) \in \mathcal{A}$ is univalent in $\mathbb{U}$, then $\|f\|<6$ and the bound 6 is sharp for the 
S. Owa, H. Saitoh, J. Sokół, M. Nunokawa - Univalency of some operators ...

Koebe function $k(z)=z /(1-z)^{2}$.

Conversely, if $f(z) \in \mathcal{A}$ satisfies $\|f\|<1$, then $f(z)$ is univalent in $\mathbb{U}$ by Becker [1]. Also, it is known that $\|f\|<4$ for $f(z) \in \mathcal{K}$. For $f(z) \in \mathcal{A}$, the Alexander transformation $J[f](z)$ is defined by

$$
J[f](z)=\int_{0}^{z} \frac{f(t)}{t} d t .
$$

If $f(z) \in \mathcal{S}$, then $f(z)$ is locally univalent and $\|J[f]\|<6$ by Kim, Choi and Sugawa [6]. Also, Yamashita [12] proved that if $f(z) \in \mathcal{S}^{*}(\alpha)$, then $\|f\|<6-4 \alpha$ and $\|J[f]\|<4(1-\alpha)$. By means of (1.5) and (1.8), we see that

$$
\{f ; z\}=\left(T_{f}(z)\right)^{\prime}-\frac{1}{2}\left(T_{f}(z)\right)^{2} .
$$

The Alexander transformation $J[f](z)$ of $f(z) \in \mathcal{A}$ is also called as Biernacki's integral. It is known that $J[f]\left(\mathcal{S}^{*}\right)=\mathcal{K}$ while $J[f](\mathcal{S})$ is not in $\mathcal{S}$. In this paper, we would like to extend the type of functions $f(z)$ to be considered by introducing a parameter $\alpha$ and setting an integral of the form

$$
F_{\alpha}(z)=\int_{0}^{z}\left(\frac{t f^{\prime}(t)}{f(t)}\right)^{\alpha} d t
$$

For more details on this integral, we refer to Goodman [4]. The following lemma due to Fukui and Sakaguchi [3] is a generalization of Jack's lemma by Jack [5] (also by Miller and Mocanu [9]).

Lemma 1.1 Let $w(z)=a_{p} z^{p}+a_{p+1} z^{p+1}+\cdots$ be analytic in $\mathbb{U}$ with $a_{p} \neq 0$ and $p \geqq 1$. If the maximum value of $|w(z)|$ on the circle $|z|=r<1$ is attained at $z=z_{0}$, then $z_{0} w^{\prime}\left(z_{0}\right) / w\left(z_{0}\right)$ is real and

$$
\frac{z_{0} w^{\prime}\left(z_{0}\right)}{w\left(z_{0}\right)} \geqq p
$$

\section{UNIVALENCY OF SOME OPERATORS}

We first derive

Theorem 2.1 Let $f(z)$ be analytic in $\mathbb{U}$ with $f(0)=0$. If $f(z)$ satisfies

$$
|f(z)| \leqq \frac{M}{1-|z|^{2}} \quad(z \in \mathbb{U})
$$


S. Owa, H. Saitoh, J. Sokół, M. Nunokawa - Univalency of some operators ...

for a bounded positive constant $M$, then

$$
|f(z)| \leqq \frac{3 \sqrt{3} M|z|}{2} \leqq \frac{3 \sqrt{3} M|z|}{2\left(1-|z|^{2}\right)} \quad\left(|z| \leqq \frac{\sqrt{3}}{3}\right)
$$

and

$$
|f(z)| \leqq \frac{\sqrt{3} M|z|}{1-|z|^{2}} \leqq \frac{3 \sqrt{3} M|z|}{2\left(1-|z|^{2}\right)} \quad\left(\frac{\sqrt{3}}{3} \leqq|z|<1\right)
$$

Proof For the case of $|z| \leqq \sqrt{3} / 3$, we have

$$
\frac{1}{1-|z|^{2}} \leqq \frac{3}{2}
$$

Thus, the inequality (2.1) gives

$$
|f(z)| \leqq \frac{3 M}{2} \quad\left(|z| \leqq \frac{\sqrt{3}}{3}\right)
$$

Therefore, applying the Schwarz lemma for $f(z)$ with $|z| \leqq \sqrt{3} / 3$, we obtain that

$$
|f(z)| \leqq \sqrt{3}|z| \frac{3 M}{2} \quad\left(|z| \leqq \frac{\sqrt{3}}{3}\right)
$$

which shows (2.2). If $\sqrt{3} / 3 \leqq|z|<1$, we know that $\sqrt{3}|z| \geqq 1$. This gives us that

$$
|f(z)| \leqq \frac{\sqrt{3} M|z|}{1-|z|^{2}} \quad\left(\frac{\sqrt{3}}{3} \leqq|z|<1\right)
$$

which implies the inequality (2.3).

Corollary 2.1 If $f(z)$ is analytic in $\mathbb{U}$ with $f(0)=0$, then there exists some $z \in \mathbb{U}$ such that

$$
|f(z)| \leqq \frac{M}{1-|z|^{2}}
$$

satisfies

$$
|f(z)| \leqq \frac{3 \sqrt{3} M|z|}{2\left(1-|z|^{2}\right)}
$$

for a positive constant $M$. 
S. Owa, H. Saitoh, J. Sokół, M. Nunokawa - Univalency of some operators ...

Remark 2.1 Noting that $3 \sqrt{3} / 2=2.598 \ldots$, we conjecture that $3 \sqrt{3} / 2$ in Corollary 2.1 can be replaced by 1 .

Next, we derive

Theorem 2.2 For a function $f(z) \in \mathcal{S}$, we assume that the function $\left(z f^{\prime}(z) / f(z)\right)^{\alpha}$ is analytic in $\mathbb{U}$ for $\alpha>0$ with

$$
\left.\left(\frac{z f^{\prime}(z)}{f(z)}\right)^{\alpha}\right|_{z=0}=1 .
$$

Then, the integral transformation $F_{\alpha}(z)$ defined by (1.12) is univalent in $\mathbb{U}$ for

$$
0<\alpha \leqq \alpha_{0}=\frac{2 \sqrt{5}-4}{15 \sqrt{3}}=0.0181725 \ldots
$$

Proof Note that

$$
F_{\alpha}^{\prime}(z)=\left(\frac{z f^{\prime}(z)}{f(z)}\right)^{\alpha} \quad(z \in \mathbb{U})
$$

by $F_{\alpha}(z)$ in (1.12). This gives us that

$$
\frac{F_{\alpha}^{\prime \prime}(z)}{F_{\alpha}^{\prime}(z)}=\frac{\alpha}{z}\left(1+\frac{z f^{\prime \prime}(z)}{f^{\prime}(z)}-\frac{z f^{\prime}(z)}{f(z)}\right) .
$$

If we put

$$
h(z)=1+\frac{z f^{\prime \prime}(z)}{f^{\prime}(z)}-\frac{z f^{\prime}(z)}{f(z)} \quad(z \in \mathbb{U}),
$$

we have that $h(0)=0$ and

$$
|h(z)| \leqq\left|1+\frac{z f^{\prime \prime}(z)}{f^{\prime}(z)}\right|+\left|\frac{z f^{\prime}(z)}{f(z)}\right| .
$$

On the other hand, it is well-known that if $f(z) \in \mathcal{S}$, then

$$
\left|\frac{z f^{\prime \prime}(z)}{f^{\prime}(z)}-\frac{2|z|^{2}}{1-|z|^{2}}\right| \leqq \frac{4|z|}{1-|z|^{2}} \quad(z \in \mathbb{U})
$$

that is,

$$
\left|1+\frac{z f^{\prime \prime}(z)}{f^{\prime}(z)}-\frac{1+|z|^{2}}{1-|z|^{2}}\right| \leqq \frac{4|z|}{1-|z|^{2}} \quad(z \in \mathbb{U}) .
$$


S. Owa, H. Saitoh, J. Sokół, M. Nunokawa - Univalency of some operators ...

This gives that

$$
\left|1+\frac{z f^{\prime \prime}(z)}{f^{\prime}(z)}\right| \leqq \frac{4|z|}{1-|z|^{2}}+\frac{1+|z|^{2}}{1-|z|^{2}}<\frac{6}{1-|z|^{2}} \quad(z \in \mathbb{U})
$$

Further, we know that

$$
\left|\frac{z f^{\prime}(z)}{f(z)}\right| \leqq \frac{1+|z|}{1-|z|}=\frac{(1+|z|)^{2}}{1-|z|^{2}}<\frac{4}{1-|z|^{2}} \quad(z \in \mathbb{U}) .
$$

Therefore, the inequality (2.15) implies that

$$
|h(z)|<\frac{10}{1-|z|^{2}} \quad(z \in \mathbb{U}) .
$$

Considering $M=10$ in (2.1) of Theorem 2.1, we say that

$$
|h(z)|<\frac{15 \sqrt{3}|z|}{1-|z|^{2}} \quad(z \in \mathbb{U}) .
$$

Therefore, we have that

$$
\left|\frac{F_{\alpha}^{\prime \prime}(z)}{F_{\alpha}^{\prime}(z)}\right| \leqq \frac{\alpha}{|z|}|h(z)|<\frac{15 \sqrt{3} \alpha}{1-|z|^{2}} \quad(z \in \mathbb{U})
$$

By using of the result in [11], we know that there exists a point $z \in \mathbb{U}$ that if

$$
|h(z)|<\frac{1}{1-|z|^{2}} \quad(z \in \mathbb{U})
$$

then

$$
\left|h^{\prime}(z)\right|<\frac{4}{\left(1-|z|^{2}\right)^{2}} \quad(z \in \mathbb{U}) .
$$

It follows from the above that

$$
\left|\left(\frac{F_{\alpha}^{\prime \prime}(z)}{F_{\alpha}^{\prime}(z)}\right)^{\prime}\right|<\frac{60 \sqrt{3} \alpha}{\left(1-|z|^{2}\right)^{2}} \quad(z \in \mathbb{U}) .
$$

Therefore, we have that

$$
\left|\left\{F_{\alpha}(z) ; z\right\}\right| \leqq\left|\left(\frac{F_{\alpha}^{\prime \prime}(z)}{F_{\alpha}^{\prime}(z)}\right)^{\prime}\right|+\frac{1}{2}\left|\frac{F_{\alpha}^{\prime \prime}(z)}{F_{\alpha}^{\prime}(z)}\right|^{2}
$$


S. Owa, H. Saitoh, J. Sokół, M. Nunokawa - Univalency of some operators ...

$$
\leqq \frac{60 \sqrt{3} \alpha}{\left(1-|z|^{2}\right)^{2}}+\frac{1}{2}\left(\frac{15 \sqrt{3} \alpha}{1-|z|^{2}}\right)^{2}=\frac{15(45 \alpha+8 \sqrt{3}) \alpha}{2\left(1-|z|^{2}\right)^{2}} \quad(z \in \mathbb{U}) .
$$

Applying the Nehari's condition (1.7) for $F_{\alpha}(z)$, we need that

$$
\frac{15(45 \alpha+8 \sqrt{3}) \alpha}{2} \leqq 2
$$

that is, that

$$
0<\alpha \leqq \alpha_{0}=\frac{2 \sqrt{5}-4}{15 \sqrt{3}}=0.0181725 \ldots
$$

This completes the proof of the theorem.

Next, we recall here a result by Chichra and Singh [2] that if

$$
z+z^{2} \log \frac{g(z)}{z} \in \mathcal{S}^{*},
$$

then there exist some $t(0 \leqq t \leqq 1)$ and $\alpha(0 \leqq \alpha \leqq 1 / 2)$ such that

$$
t z+(1-t) \int_{0}^{z}\left(\frac{t g^{\prime}(t)}{g(t)}\right)^{\alpha} d t \in \mathcal{S}^{*}
$$

Letting

$$
\frac{g(z)}{z}=\frac{z f^{\prime}(z)}{f(z)}
$$

for $f(z) \in \mathcal{A}$, Theorem 2.2 becomes

Theorem 2.3 Assume that $g(z) \in \mathcal{A}$ satisfies

$$
z \exp \left(\int_{0}^{z} \frac{\frac{g(t)}{t}-1}{t} d t\right) \in \mathcal{S},
$$

the function $(g(z) / z)^{\alpha}$ is analytic in $\mathbb{U}$ with $0<\alpha<1$ and

$$
\left.\left(\frac{g(z)}{z}\right)^{\alpha}\right|_{z=0}=1
$$

If $0<\alpha \leqq \alpha_{0}=(2 \sqrt{5}-4) / 15 \sqrt{3}=0.0181725 \cdots$, then the integration $\int_{0}^{z}(g(t) / t)^{\alpha} d t$ is univalent in $\mathbb{U}$. 
S. Owa, H. Saitoh, J. Sokół, M. Nunokawa - Univalency of some operators ...

By means of the result due to Krzyż [7], we know that $g(z) \in \mathcal{S}$ is not implies that $\int_{0}^{z}(g(t) / t) d t \in \mathcal{S}$. The counterexample for the above is given by

$$
g(z)=\frac{z}{(1-i z)^{1-i}} .
$$

On the other hand, Merkes and Wright [8] showed that if $g(z) \in \mathcal{S}^{*}$, then

$$
\int_{0}^{z}\left(\frac{g(t)}{t}\right)^{\alpha} d t \in \mathcal{C}
$$

for $-1 / 2 \leqq \alpha \leqq 3 / 2$. Theorem 2.3 says that if

$$
z \exp \left(\int_{0}^{z} \frac{\frac{g(t)}{t}-1}{t} d t\right) \in \mathcal{S}
$$

then

$$
\int_{0}^{z}\left(\frac{g(t)}{t}\right)^{\alpha} d t \in \mathcal{S}
$$

for $0<\alpha \leqq \alpha_{0}=(2 \sqrt{5}-4) / 15 \sqrt{3}$.

Corollary 2.2 If $g(z) \in \mathcal{A}$ satisfies

$$
\operatorname{Re}\left(\frac{g(z)}{z}\right)>0 \quad(z \in \mathbb{U})
$$

then

$$
\int_{0}^{z}\left(\frac{g(t)}{t}\right)^{\alpha} d t
$$

is univalent in $\mathbb{U}$, where $0<\alpha \leqq \alpha_{0}=(2 \sqrt{5}-4) / 15 \sqrt{3}$.

\section{An application of Schwarzian derivative}

Next, we would like to consider an application of Schwarzian derivative concerning with the subordinations. Let $f(z) \in \mathcal{A}$ and $g(z) \in \mathcal{A}$. Then the function $f(z)$ is said to subordinate to $g(z)$ if there exists a function $w(z)$ analytic in $\mathbb{U}$ with $w(0)=0$ and $|w(z)|<1$ such that $f(z)=g(w(z))$ for $z \in \mathbb{U}$. We write that

$$
f(z) \prec g(z) \quad(z \in \mathbb{U})
$$


S. Owa, H. Saitoh, J. Sokół, M. Nunokawa - Univalency of some operators ...

if $f(z)$ subordinates to $g(z)$ in $\mathbb{U}$. Also, if $g(z)$ is univalent in $\mathbb{U}$, then $f(z) \prec g(z)$ is equivalent to $f(0)=g(0)$ and $f(\mathbb{U}) \subset g(\mathbb{U})$ (see Miller and Mocanu [9]).

Now, we derive

Theorem 3.1 Let $f(z) \in \mathcal{A}$ satisfy

$$
\left|z^{2}\{f ; z\}\right|<\alpha(1-\beta) \quad(z \in \mathbb{U})
$$

where $0<\alpha<1$ and

$$
\left|\frac{z h^{\prime \prime}(z)}{h^{\prime}(z)}-\frac{2 z h^{\prime}(z)}{h(z)+1}\right| \leqq \beta \quad(z \in \mathbb{U})
$$

with

$$
h(z)=\left(f^{\prime}(z)\right)^{1 / \alpha} \neq \pm 1
$$

Then we have that

$$
f^{\prime}(z) \prec\left(\frac{1+z}{1-z}\right)^{\alpha} \quad(z \in \mathbb{U})
$$

or

$$
\left|\arg f^{\prime}(z)\right|<\frac{\pi}{2} \alpha \quad(z \in \mathbb{U})
$$

Therefore, $f(z)$ is univalent in $\mathbb{U}$.

Proof For $h(z)=\left(f^{\prime}(z)\right)^{1 / \alpha}(0<\alpha<1)$, we define the function $w(z)$ by

$$
w(z)=\frac{h(z)-1}{h(z)+1}=\frac{c_{n}}{2} z+\cdots
$$

with $w(0)=0$. This implies that

$$
f^{\prime}(z)=\left(\frac{1+w(z)}{1-w(z)}\right)^{\alpha} .
$$

It follows from (3.8) that

$$
f^{\prime \prime}(z)=\frac{2 \alpha w^{\prime}(z)}{1-w(z)^{2}}\left(\frac{1+w(z)}{1-w(z)}\right)^{\alpha}=\frac{2 \alpha w^{\prime}(z)}{1-w(z)^{2}} f^{\prime}(z),
$$


S. Owa, H. Saitoh, J. Sokół, M. Nunokawa - Univalency of some operators ...

that is, that

$$
\frac{f^{\prime \prime}(z)}{f^{\prime}(z)}=\frac{2 \alpha w^{\prime}(z)}{1-w(z)^{2}}
$$

Thus, we obtain that

$$
\left(\frac{f^{\prime \prime}(z)}{f^{\prime}(z)}\right)^{2}=\left(\frac{z f^{\prime \prime}(z)}{f^{\prime}(z)}\right)^{2} \frac{1}{z^{2}}=\left(\frac{2 \alpha z w^{\prime}(z)}{1-w(z)^{2}}\right)^{2} \frac{1}{z^{2}} .
$$

We suppose that there exists a point $z_{0} \in \mathbb{U}$ such that $|w(z)|<1\left(|z|<\left|z_{0}\right|<1\right)$ and $\left|w\left(z_{0}\right)\right|=1$. Then Lemma 1.1 gives us that

$$
\frac{z_{0} w^{\prime}\left(z_{0}\right)}{w\left(z_{0}\right)}=k \geqq 1 \text {. }
$$

Further, by the result due to Miller and Mocanu [9], we have that

$$
\operatorname{Re}\left(\frac{z_{0} w^{\prime \prime}\left(z_{0}\right)}{w^{\prime}\left(z_{0}\right)}\right) \geqq 0
$$

Therefore, we have that

$$
\begin{aligned}
& \left(\frac{f^{\prime \prime}\left(z_{0}\right)}{f^{\prime}\left(z_{0}\right)}\right)^{2}=\left(\frac{2 \alpha k w\left(z_{0}\right)}{1-w\left(z_{0}\right)^{2}}\right)^{2} \frac{1}{z_{0}^{2}} \\
& =\left(\frac{i \alpha k}{\sin \theta}\right)^{2} \frac{1}{z_{0}^{2}}=-\left(\frac{\alpha k}{\sin \theta}\right)^{2} \frac{1}{z_{0}^{2}},
\end{aligned}
$$

where $w\left(z_{0}\right)=e^{i \theta}(0 \leqq \theta<2 \pi)$.

Also, we see that

$$
\begin{aligned}
& \left.\left(\frac{f^{\prime \prime}(z)}{f^{\prime}(z)}\right)^{\prime}\right|_{z=z_{0}}=\left.\left(\frac{2 \alpha w^{\prime}(z)}{1-w(z)^{2}}\right)^{\prime}\right|_{z=z_{0}} \\
= & 2 \alpha\left(\frac{w^{\prime \prime}\left(z_{0}\right)}{1-w\left(z_{0}\right)^{2}}\right)+\left.\frac{4 \alpha w(z)\left(w^{\prime}(z)\right)^{2}}{\left(1-w(z)^{2}\right)^{2}}\right|_{z=z_{0}} \\
= & \frac{i k \alpha}{\sin \theta}\left(\frac{z_{0} w^{\prime \prime}\left(z_{0}\right)}{w^{\prime}\left(z_{0}\right)}\right) \frac{1}{z_{0}}+\left(\frac{i k}{\sin \theta}\right)^{2} \frac{\alpha w\left(z_{0}\right)}{z_{0}^{2}} \\
= & \frac{k \alpha}{\sin \theta}\left\{i\left(\frac{z_{0} w^{\prime \prime}\left(z_{0}\right)}{w^{\prime}\left(z_{0}\right)}\right)-\frac{k w\left(z_{0}\right)}{\sin \theta}\right\} \frac{1}{z_{0}^{2}} .
\end{aligned}
$$


S. Owa, H. Saitoh, J. Sokół, M. Nunokawa - Univalency of some operators ...

Consequetly, we obtain that

$$
\begin{aligned}
& z_{0}^{2}\{f ; z\}=\frac{k \alpha}{\sin \theta}\left\{i\left(\frac{z_{0} w^{\prime \prime}\left(z_{0}\right)}{w^{\prime}\left(z_{0}\right)}\right)-\frac{k w\left(z_{0}\right)}{\operatorname{sim} \theta}+\frac{\alpha k}{2 \sin \theta}\right\} \\
= & \frac{k \alpha}{2 \sin \theta}\left\{2 i\left(\frac{z_{0} w^{\prime \prime}\left(z_{0}\right)}{w^{\prime}\left(z_{0}\right)}\right)+\frac{k}{\sin \theta}(\alpha-2 \cos \theta-2 i \sin \theta)\right\}
\end{aligned}
$$

and so

$$
\begin{aligned}
&\left|z_{0}^{2}\left\{f ; z_{0}\right\}\right| \geqq \frac{\alpha}{2}|| \frac{k}{\sin \theta}|| \alpha-2 \cos \theta-2 i \sin \theta|-2| \frac{z_{0} w^{\prime \prime}\left(z_{0}\right)}{w^{\prime}\left(z_{0}\right)}|| \\
& \geqq \frac{\alpha}{2}\left|k \sqrt{\frac{\alpha^{2}-4 \alpha \cos \theta+4}{1-\cos ^{2} \theta}}-2\right| \frac{z_{0} w^{\prime \prime}\left(z_{0}\right)}{w^{\prime}\left(z_{0}\right)}|| .
\end{aligned}
$$

If we define a function $p(x)$ by

$$
p(x)=\frac{\alpha^{2}-4 \alpha x+4}{1-x^{2}} \quad(x=\cos \theta)
$$

then

$$
p^{\prime}(x)=\frac{-2(2 x-\alpha)(\alpha x-2)}{\left(1-x^{2}\right)^{2}}
$$

gives that $p(x)$ takes its minimum value at $x=\alpha / 2<1 / 2$, because $0<\alpha<1$ and $-1 \leqq x \leqq 1$. This shows us that $p(x) \geqq 4$ and so

$$
\begin{gathered}
\left|z_{0}^{2}\left\{f ; z_{0}\right\}\right| \geqq \alpha|1-| \frac{z_{0} w^{\prime \prime}\left(z_{0}\right)}{w^{\prime}\left(z_{0}\right)}|| \\
=\alpha|1-| \frac{z_{0} h^{\prime \prime}\left(z_{0}\right)}{h^{\prime}\left(z_{0}\right)}-\frac{2 z_{0} h^{\prime}\left(z_{0}\right)}{h\left(z_{0}\right)+1}|| \geqq \alpha(1-\beta) .
\end{gathered}
$$

This contradicts the condition (3.2) of the theorem. Therefore, there is no $z_{0} \in \mathbb{U}$ such that $\left|w\left(z_{0}\right)\right|=1$. This implies that there exists $w(z)$ such that

$$
f^{\prime}(z)=\left(\frac{1+w(z)}{1-w(z)}\right)^{\alpha} \quad(z \in \mathbb{U})
$$

with $w(0)=0$ and $|w(z)|<1(z \in \mathbb{U})$. Consequently, we prove the subordination (3.5).

Further, since

$$
\left|\arg \left(\frac{1+z}{1-z}\right)\right|<\frac{\pi}{2} \quad(z \in \mathbb{U}),
$$


S. Owa, H. Saitoh, J. Sokół, M. Nunokawa - Univalency of some operators ...

we obtain (3.6) for $\arg f^{\prime}(z)$.

Making $\alpha=1 / 2$ in Theorem 3.1, we derive

Corollary 3.1 Let $f(z) \in \mathcal{A}$ satisfy

$$
\left|z^{2}\{f ; z\}\right|<\frac{1-\beta}{2} \quad(z \in \mathbb{U})
$$

with

$$
\left|\frac{z h^{\prime \prime}(z)}{h^{\prime}(z)}-\frac{2 z h^{\prime}(z)}{h(z)+1}\right| \leqq \beta \quad(z \in \mathbb{U})
$$

and $h(z)=\sqrt{f^{\prime}(z)} \neq \pm 1$. Then we have

$$
f^{\prime}(z) \prec \sqrt{\frac{1+z}{1-z}} \quad(z \in \mathbb{U})
$$

or

$$
\left|\arg f^{\prime}(z)\right|<\frac{\pi}{4} \quad(z \in \mathbb{U})
$$

Acknowledgements Professor Mamoru Nunokawa has got the decoration of $\lceil Z$ uihouchujishou」 by Japanese Gavernment on May 12, 2017. He would like to express his deep thanks for Prof. Owa, Prof. Saitoh and Prof. Sokółto support his research for long time.

\section{REFERENCES}

[1] J. Becker, Löwnersche Differentialgleichung und quasikonform fortsetzbare schlichte Funktionen (German), J. Reine Angew. Math., 255(1972), 23 - 43.

[2] P. N. Chichra and R. Singh, Convex sum of univalent functions. J. Austral. Math. Soc., 14(1972), $503-507$.

[3] S. Fukui and K. Sakaguchi, An extension of a theorem of S. Ruscheweyh, Bull. Fac. Edu. Wakayama Univ. Nat. Sci., 29(1980), 1-3.

[4] A. W. Goodman, Univalent Functions, Vols. I and II, Mariner Publishing Co., Tampa, Florida (1983).

[5] I. S. Jack, Functions starlike and convex of order $\alpha$, J. London Math. Soc., 3(1971), 469-474. 
S. Owa, H. Saitoh, J. Sokół, M. Nunokawa - Univalency of some operators ...

[6] Y.C. Kim, J.H. Choi and T. Sugawa, Coefficient bounds and convolution properties for certain classes of close-to-convex functions. Proc. Japan Acad., 76(2000), $95-98$.

[7] J. Krzyż and Z. Lewandowski, On the integral of univalent functions, Bull. Acad. Polon. Sci. Sér. Sci. Math. Astronom. Phys., 11(1963), 447- 448.

[8] E. P. Merkes and D. J. Wright, On the univalence of a certain integral, Proc. Amer. Math. Soc., 27(1971), 97 - 100.

[9] S. S. Miller and P. T. Mocanu, Differential Subordinations, Theory and Applications, Series of Monographs and Textbooks in Pure and Applied Mathematics, Vol. 225, Marcel Dekker Inc., New York / Basel 2000.

[10] Z. Nehari, The Schwarzian derivative and schlicht functions, Bull. Amer. Math. Soc., 55(1949), 545 - 551.

[11] Research Problems, Bull. Amer. Math. Soc., 71(1965), 545 - 551. Research Problems from a Symposium on Analytic Function Theory which was held at the University of Kentucky, May 28-June 1, 1965.

[12] S. Yamashita, Norm estimates for function starlike or convex of order alpha. Hokkaido Math. J., 28(1999), 217 - 230.

Shigeyoshi Owa

Department of Mathematics

Yamato University

Katayama 2-5-1, Suita, Osaka 564-0082, Japan

e-mail: shige21@ican.zaq.ne.jp

Hitoshi Saitoh

Department of Mathematics

Gunma National College of Technology

Toriba, Maebashi, Gunma 371-8530, Japan

e-mail: sp822457@db4.so-net.ne.jp

Janusa Sokół

University of Rzeszów

Faculty of Mathematics and Natural Sciences

UL. Prof. Pigonia 1, 35-310 Rzeszów, Poland

e-mail: jsokol@ur.edu.pl

Mamoru Nunokawa

Honorary Professor 
S. Owa, H. Saitoh, J. Sokół, M. Nunokawa - Univalency of some operators ...

University of Gunma

Hoshikuki-Cho 798-8, Chuou-Ward, Chiba 260-0808, Japan

e-mail:mamoru_nuno@doctor.nifty.jp 